\title{
Kinetics of Transesterification of Safflower Oil to Obtain Biodiesel Using Heterogeneous Catalysis
}

\section{DOI 10.1515/ijcre-2015-0108}

\begin{abstract}
The kinetics of the transesterification of safflower oil and methanol catalyzed by $\mathrm{K}_{2} \mathrm{O} / \mathrm{NaX}$ was studied and modeled. The influence of the oil-methanol initial molar ratio and amount of catalyst were investigated to achieve a maximum triglycerides conversion ( $99 \%$ ) and a final methyl esters content of $94 \% \pm 1$. A kinetic model based on an EleyRideal mechanism was found to best fit the experimental data when assuming methanol adsorption as determining step. Other models derived from Langmuir - Hinshelwood Hougen -Watson (LHHW) mechanisms were rejected based on statistical analysis, mechanistic considerations and physicochemical interpretation of the estimated parameters.
\end{abstract}

Keywords: potassium nitrate, potassium oxide, Kinetics, Safflower oil, Transesterification, Zeolite

\section{Introduction}

Biodiesel is defined as monoalkyl esters of fatty acids derived from vegetable oils or animal fats. It can be burnt as petroleum diesel so it can be used without major engine modifications (Demirbas 2008a). Albeit vegetable oils may be used directly in the engine, their previous

*Corresponding authors: Rubi Romero, Reyna Natividad, Centro Conjunto de Investigación en Química Sustentable UAEM-UNAM. Carretera Toluca-Atlacomulco Km 14.5, Unidad San Cayetano, Toluca Estado de México, 50200, México, E-mail: rromeror@uaemex.mx, reynanr@gmail.com

Gabriel E. Galván Muciño, Centro Conjunto de Investigación en Química Sustentable UAEM-UNAM. Carretera Toluca-Atlacomulco Km 14.5, Unidad San Cayetano, Toluca Estado de México, 50200, México http://orcid.org/0000-0003-3692-2069

Armando Ramírez, Facultad de Química, Universidad Autónoma del Estado de México, Paseo Colón esq. Paseo Tollocan s/n 50120 Toluca, Estado de México, México

María Jesús Ramos, Departamento de Ingeniería Química, Instituto de Tecnologías Química y Medioambiental, Universidad de Castilla-La Mancha, Avd. Camilo José Cela s/n, 13071 Ciudad Real, Spain Ramiro Baeza-Jiménez, Centro Conjunto de Investigación en Química Sustentable UAEM-UNAM. Carretera Toluca-Atlacomulco Km 14.5, Unidad San Cayetano, Toluca Estado de México, 50200, México transesterification to obtain biodiesel is desirable to decrease their viscosity and fulfill international standards for petroleum diesel (Demirbas 2008b). This process consists basically of three-steps, in which one mole of triglyceride (T) reacts with one mole of alcohol to form one mole of diglyceride (D), which then reacts with other mole of alcohol to form one mole of monoglyceride $(\mathrm{M})$ and finally this reacts with a third mole of alcohol to form one mole of glycerol (G). In each step one mole of alkyl ester (E) is formed. The preferred alcohol to conduct the transesterification of vegetable oils is methanol due to its low price and high reactivity (Meneghetti et al. 2007, Zhou and Boocock 2006). The reaction scheme for transesterification reaction is shown in Figure 1.

The transesterification reaction can be performed in either acidic or basic medium. The latter occurs in the presence of a homogeneous catalyst such as sodium hydroxide or potassium hydroxide. Currently the industrial production of biodiesel is mainly conducted using this kind of catalyst in a batch reactor. An alternative is the use of basic heterogeneous catalysts. This heterogeneous process implies advantages such as reduction on the use of water to purify products, relatively easy separation of reactants and products and decrease of the use of specialized corrosion equipment. In addition, heterogeneous catalysts are reusable and have a lower environmental impact (Zhang et al. 2010, Cao et al. 2008). Actually, high methyl esters yields (>90\%) have been obtained with heterogeneous basic catalysts like $\mathrm{K}_{2} \mathrm{O}$, $\mathrm{BaO}, \mathrm{CaO}, \mathrm{SrO}, \mathrm{MgO}$ and $\mathrm{La}_{2} \mathrm{O}$ (Kawashima, Matsubara, and Honda 2009, Granados et al. 2009, Zabeti, Wan Daud, and Aroua 2009, Venkat Reddy, Oshel, and Verkade 2006, Peña et al. 2013). In this context, mass transport resistances have been reported to be negligible with stirring speeds between 200 and 1,500 rpm (Froment, Bischoff and De Wilde, 2011, Van de Steene, De Clercq, and Thybaut 2012, Kim et al. 2004). Also, it is worth pointing out that in order to overcome the mixing problems, the use of co-solvents like $n$-hexane, has been proven to enhance up to $10 \%$ the biodiesel yield (Kim et al. 2004). Albeit $\mathrm{CaO}$ has been extensively used due to its long life and requirement of mild reaction conditions (Math, Kumar, and Chetty 2010), the $\mathrm{K}_{2} \mathrm{O} / \mathrm{NaX}$ is emerging as a catalytic system with great potential since has been reported 
$T+R-O H \rightleftharpoons D+E$

$\mathrm{D}+\mathrm{R}-\mathrm{OH} \rightleftharpoons \mathrm{M}+\mathrm{E}$

$M+R-O H \rightleftharpoons G+E$

Figure 1: Transesterification reaction scheme.

to exhibit a high catalytic activity (98.2\% methyl esters content) in the transesterification reaction of sunflower oil (Peña et al. 2013). This is actually the catalyst that was studied in this work and synthesized by impregnating the catalytic support ( $\mathrm{NaX}$ zeolite) with an aqueous potassium nitrate solution (Peña et al. 2013). In order to understand how this catalyst works and to further apply it, at industrial level for instance, a kinetic model is desirable. In this sense, it is to the authors knowledge that none kinetic model has been reported for this catalyst. Therefore, this work focuses on the development of a kinetic model to describe the reaction of safflower with methanol to produce biodiesel by using $\mathrm{K}_{2} \mathrm{O}$ supported on $\mathrm{NaX}$ zeolite as catalyst. To achieve so, several mechanisms were proposed and a discrimination process was applied to the resulting rate equations. The mechanisms were suggested based upon previous experiences with other catalysts (Lee, Park, and Lee 2009, Câmara and Aranda 2011, Hattori, Shima, and Kabashima 2000, Kapil et al. 2011, Dossin, Reyniers, and Marin 2006). In this context and generally speaking, the models that have been proven to better describe the kinetic behavior of the transesterification reaction of vegetable oils catalyzed by basic heterogeneous compounds, have been mainly based on LangmuirHinshelwood-Hougen-Watson (LHHW), Eley-Rideal (ER) and Hattori assumptions (Hattori, Shima, and Kabashima 2000, Kapil et al. 2011, Dossin, Reyniers, and Marin 2006). In addition to the type of mechanism, what has been found to be an important variable is the limiting step. In some cases it has been reported that the adsorption of methanol is the limiting step and in other cases the surface reaction has been identified as the slowest step in the transesterification process (Lee, Park, and Lee 2009, Câmara and Aranda 2011, Dossin, Reyniers, and Marin 2006). Both, the mechanism and limiting step mainly depend on the employed catalyst and thus is important to elucidate them for the $\mathrm{K}_{2} \mathrm{O} / \mathrm{NaX}$ catalytic system.

\section{Materials and methods}

\subsection{Materials}

Refined safflower oil employed for the transesterification reaction was purchased from a local grocery store.
The viscosity, acid value and water content of the oils were determined according to the European Union Quality Standard (EN-14214). According to this method, the oil viscosity at $40^{\circ} \mathrm{C}$ is $39.25 \mathrm{~mm}^{2} / \mathrm{s}$, the acid value is $0.11 \mathrm{mg} \mathrm{KOH} / \mathrm{g}$ and the water content is 0.05 wt $\%$. Anhydrous methanol (HPLC $99.9 \%, \mathrm{H}_{2} \mathrm{O}<0.02$ ) and potassium nitrate (ACS 99.3\%) were supplied by Fermont. A NaX zeolite powder with 13X molecular sieve and average particle size of $2 \mu \mathrm{m}$ and molar ratio Si/Al of 1:23 obtained from Sigma-Aldrich was used as catalytic support. The gas chromatography reference standard for fatty methyl esters was obtained from Supelco. Methyl heptadecanoate (puriss. p.a., standard for GC, $\geq 99.7 \%$ ), Tricaprin, Glyceryl trioleate, 1,3Diolein and Monoolein ( $\geq 99 \%)$ were supplied by Sigma-Aldrich.

\subsection{Catalyst preparation}

As mentioned within introduction, the catalytic support (NaX zeolite) was impregnated with an aqueous potassium nitrate solution. Prior impregnation, the zeolite was calcined at $500^{\circ} \mathrm{C}$ under flowing air at a rate of $4^{\circ} \mathrm{C} / \mathrm{min}$ and held at this temperature for $10 \mathrm{~h}$ to eliminate any adsorbed water on the zeolitic surface. For the same purpose, the zeolite was placed in a glass vessel and kept under vacuum at room temperature for $2 \mathrm{~h}$. An aqueous $\mathrm{KNO}_{3}$ solution of known concentration was then poured over the zeolite. The solvent was removed by evaporation under vacuum. The concentration of impregnation solution was calculated to always yield a final potassium content of $13.5 \mathrm{wt} \%$. After impregnation the catalysts were dried at $120^{\circ} \mathrm{C}$ overnight and calcined at a rate of $1{ }^{\circ} \mathrm{C} / \mathrm{min}$ at $500^{\circ} \mathrm{C}$ and kept at this temperature during $4 \mathrm{~h}$ to obtain potassium oxide. The characterization of the prepared catalyst has been previously reported (Peña et al. 2013) and therefore was not included in this paper.

\subsection{Transesterification reaction}

Biodiesel synthesis was carried out in a $250 \mathrm{ml}$ flat bottom cylindrical reactor, equipped with baffles, a condensation system and magnetic stirring (900 rpm). The reaction volume was $150 \mathrm{ml}$. The stirring speed was maximized in order to reduce the solid-liquid mass transfer resistance. In addition, the use of a co-solvent was not considered mandatory since the mixing problems were overcome by the use of baffles and the stirring speed. All experiments were carried out at atmospheric pressure, temperature of 
$60^{\circ} \mathrm{C} \pm 1{ }^{\circ} \mathrm{C}$ during $7 \mathrm{~h}$. These conditions were elected based upon previous studies (Peña 2009). After heating the oil up to the reaction temperature, the alcohol and catalyst were added and mixed to start the transesterification reaction. Multiple experiments were performed modifying methanol:oil molar ratio (6:1, 12:1, 15:1, 18:1, 21:1 and 24:1) and catalyst concentration referred to the initial oil weight $(10,12.5,15 \mathrm{wt} \%)$. At the end of the reaction the catalyst was separated from the products (glycerin and methyl esters) by centrifugation. The residual methanol was separated via rotary evaporation.

Samples $(1.5 \mathrm{ml})$ were taken at different reaction times, cooled down with ice and the catalyst was separated from the products and reactants by centrifugation at 13,000 rpm during $5 \mathrm{~min}$ and returned to the reactor. The supernatant was analyzed by gas chromatography to obtain the experimental data to prepare the kinetic model.

\subsection{Chromatographic analysis}

The concentration of all species involved in the reactions was determined by gas chromatography using a Varian 3800 chromatograph equipped with a CP-8410 auto injector and a flame ionization detector (FID). The capillary column was an Agilent HP-Innowax column with a length of $30 \mathrm{~m}$, a film thickness of $0.25 \mu \mathrm{m}$ and an internal diameter of 0.32 $\mathrm{mm}$. The analysis was performed according to EN-14103 standard. The content of glycerides, glycerol included, was conducted with an Agilent DB5-HT non-polar column with a length of $15 \mathrm{~m}$, a film thickness of $0.10 \mu \mathrm{m}$ and an internal diameter of $0.32 \mathrm{~mm}$. Helium was used as the carrier gas for both methyl esters and glycerides determinations. The type of injector and heating ramps were adapted from EN-14105 standard due to the high concentration of triglycerides at the beginning of the reaction. The analysis was carried out under conditions summarized in Table 1.

Table 1: Chromatographic analysis parameters.

\begin{tabular}{ll}
\hline Parameter & Condition \\
\hline Injector & Split/Splitless \\
Split ratio & 100 \\
Pressure & $10 \mathrm{psi}$ \\
Injector temperature & $360^{\circ} \mathrm{C}$ \\
Oven program & $120^{\circ} \mathrm{C}$ for 5 min \\
& $15^{\circ} \mathrm{C} / \mathrm{min}$ to $180^{\circ} \mathrm{C}$ \\
& $7^{\circ} \mathrm{C} / \mathrm{min}$ to $230^{\circ} \mathrm{C}$ \\
& $10 \mathrm{C} / \mathrm{min}$ to $360^{\circ} \mathrm{C}$ \\
Detector temperature & $360^{\circ} \mathrm{C}$ \\
\hline
\end{tabular}

\section{Modeling and regression analysis}

The reaction rate was considered to follow the expression,

$$
\frac{d n_{\text {FAME }}}{d t}=r^{\prime} W
$$

where $n_{\text {FAME }}$ is the number of moles of methyl esters, $W$ is the mass of catalyst and $r^{\prime}$ the reaction rate per mass of catalyst. Kinetic parameters were estimated through the minimization of the objective function,

$$
S^{2}=\frac{\sum\left(r_{i}^{\prime}-\widehat{r}_{i}^{\prime}\right)^{2}}{(n-p)}
$$

Where $\sum\left(r_{i}^{\prime}{ }_{i} \widehat{r}_{i}\right)^{2}$ is the residual sum of the squares errors for the $\mathrm{i}^{\text {th }}$ data point obtained experimentally and with the model, $n$ is the number of data elements and $p$ is the number of parameters in the model. The minimization of the objective function was achieved by LevenbergMarquardt algorithm. The parameter values were accepted or rejected based on their physico-chemical and statistical significances. The latter was established based on their individual $t$-values. Discrimination between two rival models, A and B, was done by calculating the ratio of the corresponding residual sum of squares, divided each by their degrees of freedom,

$$
P_{A, B}=\frac{\frac{\chi_{A}{ }^{2}}{\left(n-p_{A}\right)}}{\frac{\chi_{B}{ }^{2}}{\left(n-p_{B}\right)}}
$$

if this criterion ratio is smaller than 1 than model $\mathrm{A}$ describes better the experimental results than model B (Kapil et al. 2011).

\section{Results and discussion}

\subsection{Effect of methanol/oil molar ratio}

The methanol/oil molar ratio is a determining factor of the efficiency of the transesterification reaction (Gerpen 2005, Meher, Vidya Sagar, and Naik 2006). The reaction stoichiometry is 3:1 to produce 3 moles of methyl esters (biodiesel) and $1 \mathrm{~mol}$ of glycerol. However, this reaction is reversible and is controlled by chemical equilibrium, which can be shifted forwards by increasing the oil-methanol molar ratio. In addition, it should be considered that methanol is not soluble in oil and therefore, under the presence of a heterogeneous catalyst, three phases will be formed at the 
beginning of the reaction. This may limit the contact of the reactants with the catalyst. Therefore, the use of an excess of methanol is advised in order to reach the highest methyl esters content. In this work, the essayed methanol to oil molar ratios were 6:1, 12:1, 15:1, 18:1, 21:1 and 24:1 (methanol:oil). To study this variable the catalyst concentration referred to the initial oil weight was $10 \mathrm{wt} \%$ and the maximum obtained methyl esters content was around $89 \%$ with no significant effect of the initial molar alcohol: oil ratio $(p>0.05)$ in the range of 18:1 to 24:1. The methyl esters content was $50 \%, 80 \%$ and $84 \%$ for the methanol: oil molar ratios of 6:1,12:1 and 15:1; accordingly. The obtained similar contents can be explained by the optimal stirring speed $(900 \mathrm{rpm})$ and the presence of baffles in the employed reactor. Baffles promote mixing of methanol with oil and contact of the reagents with the catalyst avoiding mass transfer limitation. Due to this, the lowest molar ratio was selected (18:1) for further experimentation and kinetic modelling.

\subsection{Effect of catalyst loading}

The effect of this variable was explored at a molar ratio of 18:1 (methanol:oil), $60^{\circ} \mathrm{C}$ and $900 \mathrm{rpm}$, during $7 \mathrm{~h}$. Three different catalyst concentrations were tested, $10 \%, 12.5 \%$ and $15 \%$ respect to the initial mass of safflower oil. The content of methyl esters is listed in Table 2. It can be observed that increasing the amount of catalyst from 10 to $12.5 \%$ has a significant effect on methyl esters content $(p<0.05)$. This suggests the non existence of liquid-solid mass transport resistances. It can also be observed in Table 2 and in Figure 2 that a further increase on catalyst loading up to $15 \%$, does not lead to a significant improvement on methyl esters content. This may be due to an increase in viscosity of the reaction mixture that increases the mass transfer resistance in the multiphase system (Kim et al. 2004). Furthermore, when increasing

Table 2: Effect of catalyst loading on the final methyl esters content. Reaction conditions: molar ratio of $18: 1$ (methanol:oil), $T=60^{\circ} \mathrm{C}$, reaction time $=7 \mathrm{~h}$.

\begin{tabular}{lrrr}
\hline Experiment number & \multicolumn{2}{c}{ Catalyst loading } \\
\cline { 2 - 4 } & $\mathbf{1 0}$ \% cat/oil & $\mathbf{1 2 . 5} \%$ cat/oil & $\mathbf{1 5 \%}$ cat/oil \\
\hline 1 & 87.7 & 93.1 & 94.5 \\
2 & 89.4 & 94.0 & 96.2 \\
3 & 91.2 & 95.3 & 94.9 \\
4 & 90.3 & 93.8 & 95.3 \\
Mean & 89.6 & 94.1 & 95.2 \\
\hline
\end{tabular}

catalyst concentration the accumulation of catalyst in the deflectors was observed. This does not occur with catalyst loading $<12.5 \%$ and that is why it was selected as the optimal catalyst concentration obtaining a $94 \%$ methyl ester content in final product, with a selectivity to methyl esters of 0.97 of initial triglycerides.

\subsection{Kinetic modelling}

Two types of mechanisms were studied, LHHW and ER. For each mechanism, several rate equations were deduced depending on the rate-determining step that was postulated. For each mechanism we considered the cases with multiple surface reactions for the formation of intermediate compounds (9 elementary steps LHHW mechanism and 7 elementary steps ER mechanism) and without these intermediate steps (5 elementary steps LHHW mechanism and 3 elementary steps ER mechanism). Therefore, 4 mechanisms were proposed. For each mechanism several reaction rates expressions were established depending on the number of elementary steps, i. e. every reaction rate in Table 3 was obtained by assuming that the elementary step on the left was the rate governing step. Thus, the rate determining elementary steps and the accordingly established models are presented in Table 3(a)-(d).

The LHHW mechanism was established by assuming that both, the methanol $(\mathrm{Me})$ and triglycerides $(T)$, adsorb onto active sites with the same characteristics, followed by the surface reaction between adsorbed triglycerides $\left(T^{*}\right)$ with adsorbed methanol $\left(M e^{*}\right)$ to form adsorbed diglycerides $\left(D^{*}\right)$ and adsorbed methyl esters $\left(E^{*}\right)$. Subsequently, adsorbed methanol reacts with adsorbed diglycerides $\left(D^{*}\right)$ to form adsorbed monoglycerides $\left(M^{*}\right)$ and adsorbed methyl esters $\left(E^{*}\right)$. Finally adsorbed monoglycerides $\left(M^{*}\right)$ reacts with adsorbed methanol $\left(M e^{*}\right)$ to form adsorbed glycerol $\left(G^{*}\right)$ along with adsorbed methyl esters $\left(E^{*}\right)$. Glycerol and methyl esters are then desorbed from the catalytic surface ( $G$ and $E$ ).

The ER mechanism assumes methanol $(\mathrm{Me})$ adsorption in an empty active catalyst site, followed by the surface reaction between the adsorbed methanol $\left(\mathrm{Me}^{*}\right)$ and triglycerides $(T)$ from the bulk to form adsorbed diglycerides $\left(D^{*}\right)$ that desorb from the catalytic surface $(D)$ and react with other adsorbed methanol $\left(M e^{*}\right)$ and form adsorbed monoglycerides $\left(M^{*}\right)$ that repeat the previous step to form adsorbed glycerol $\left(G^{*}\right)$. It has been considered that in every step a methyl ester is formed $(E)$ and finally the glycerol desorb from the catalytic surface $(G)$. At all models, in the case of the elementary steps of 


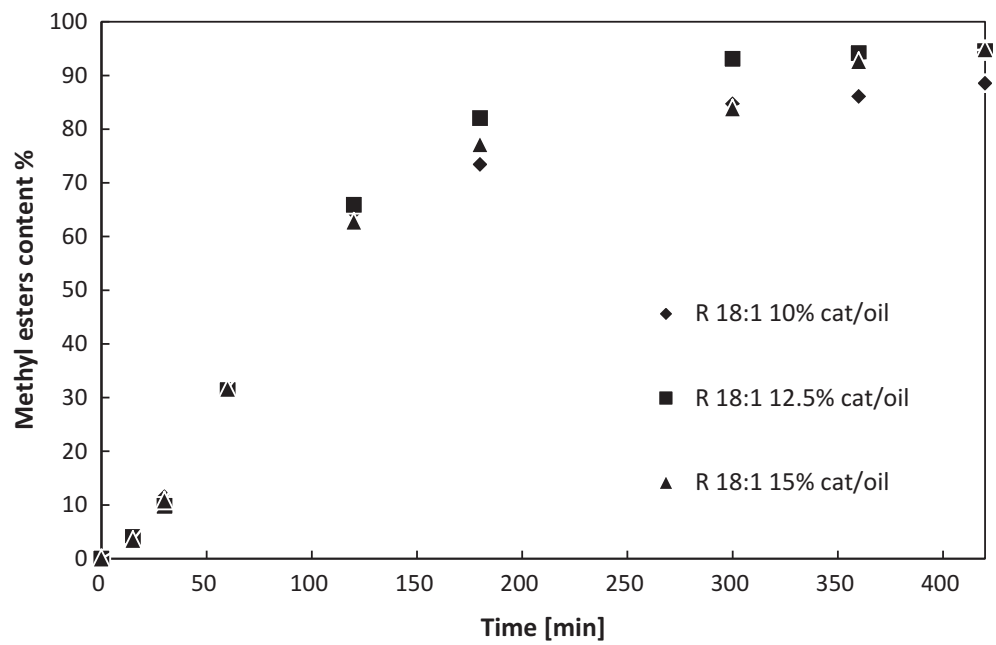

Figure 2: Effect of catalyst loading on methyl esters content: $10 \%, 12.5 \%$ and $15 \%$ (cat/oil). Reaction conditions: 18:1 (methanol:oil molar ratio), $60^{\circ} \mathrm{C}$ and $900 \mathrm{rpm}$.

Table 3: Kinetic models.

Rate determining elementary step

(a) Based on 9 elementary steps LHHW mechanism

$\mathrm{Me}+{ }^{*} \rightleftharpoons M e^{\star}$

$T+^{*} \rightleftharpoons T^{\star}$

$M e^{\star}+T^{\star} \rightleftharpoons D^{\star}+F^{\star}$

$M e^{\star}+D^{\star} \rightleftharpoons M^{\star}+F^{\star}$

$M e^{\star}+M^{\star} \rightleftharpoons G^{\star}+F^{\star}$

$D^{\star} \rightleftharpoons D+*$

$M^{\star} \rightleftharpoons M+*$

$G^{*} \rightleftharpoons G+*$

$F^{\star} \rightleftharpoons F+*$

(b) Based on 5 elementary steps LHHW mechanism

$\mathrm{Me}+{ }^{*} \rightleftharpoons M e^{\star}$

$T+^{*} \rightleftharpoons T^{\star}$
Model

$r_{1}=\frac{\mathrm{k}_{1}\left(M e-\frac{\mathrm{K}_{6} \mathrm{~K}_{9} D F}{\mathrm{~K}_{1} \mathrm{~K}_{2} \mathrm{~K}_{3} T}\right)}{1+\frac{\mathrm{K}_{6} \mathrm{~K}_{9} D F}{\mathrm{~K}_{2} \mathrm{~K}_{3} T}+\mathrm{K}_{2} T+\mathrm{K}_{6} D+\mathrm{K}_{7} M+\mathrm{K}_{8} G+\mathrm{K}_{9} F}$

$r_{2}=\frac{\mathrm{k}_{2}\left(T-\frac{\mathrm{K}_{6} \mathrm{~K}_{9} D F}{\mathrm{~K}_{1} \mathrm{~K}_{2} \mathrm{~K}_{3} M e}\right)}{1+\mathrm{K}_{1} M e+\frac{\mathrm{K}_{6} \mathrm{~K}_{9} D F}{\mathrm{~K}_{1} \mathrm{~K}_{3} M e}+\mathrm{K}_{6} D+\mathrm{K}_{7} M+\mathrm{K}_{8} G+\mathrm{K}_{9} F}$

$r_{3}=\frac{\mathrm{k}_{3}\left(\mathrm{~K}_{1} \mathrm{~K}_{2} M e T-\frac{\mathrm{K}_{6} \mathrm{~K}_{9} D F}{\mathrm{~K}_{3}}\right)}{\left(1+\mathrm{K}_{1} M e+\mathrm{K}_{2} T+\mathrm{K}_{6} D+\mathrm{K}_{7} M+\mathrm{K}_{8} G+\mathrm{K}_{9} F\right)^{2}}$

$r_{4}=\frac{\mathrm{k}_{4}\left(\mathrm{~K}_{1} \mathrm{~K}_{6} M e D-\frac{\mathrm{K}_{7} \mathrm{~K}_{9} M F}{\mathrm{~K}_{4}}\right)}{\left(1+\mathrm{K}_{1} M e+\mathrm{K}_{2} T+\mathrm{K}_{6} D+\mathrm{K}_{7} M+\mathrm{K}_{8} G+\mathrm{K}_{9} F\right)^{2}}$

$r_{5}=\frac{\mathrm{K}_{5}\left(\mathrm{~K}_{1} \mathrm{~K}_{7} M e M-\frac{\mathrm{K}_{8} \mathrm{~K}_{9} G F}{\mathrm{~K}_{5}}\right)}{\left(1+\mathrm{K}_{1} M e+\mathrm{K}_{2} T+\mathrm{K}_{6} D+\mathrm{K}_{7} M+\mathrm{K}_{8} G+\mathrm{K}_{9} F\right)^{2}}$

$r_{6}=\frac{\mathrm{K}_{-6}\left(\frac{\mathrm{K}_{7} \mathrm{~K}_{9} M F}{\mathrm{~K}_{1} \mathrm{~K}_{4} M e}-\mathrm{K}_{6} D\right)}{1+\mathrm{K}_{1} M e+\mathrm{K}_{2} T+\frac{\mathrm{K}_{7} \mathrm{~K}_{9} M F}{\mathrm{~K}_{1} \mathrm{~K}_{4} M e}+\mathrm{K}_{7} M+\mathrm{K}_{8} G+\mathrm{K}_{9} F}$

$r_{7}=\frac{\mathrm{K}_{-7}\left(\frac{\mathrm{K}_{8} \mathrm{~K}_{9} G F}{\mathrm{~K}_{1} \mathrm{~K}_{5} M e}-\mathrm{K}_{7} M\right)}{1+\mathrm{K}_{1} M e+\mathrm{K}_{2} T+\mathrm{K}_{6} D+\frac{\mathrm{K}_{7} \mathrm{~K}_{9} G F}{\mathrm{~K}_{1} \mathrm{~K}_{5} M e}+\mathrm{K}_{8} G+\mathrm{K}_{9} F}$

$r_{8}=\frac{\mathrm{K}_{-8}\left(\frac{\mathrm{K}_{1} \mathrm{~K}_{5} \mathrm{~K}_{7} M e M}{\mathrm{~K}_{9} F}-\mathrm{K}_{8} G\right)}{1+\mathrm{K}_{1} M e+\mathrm{K}_{2} T+\mathrm{K}_{6} D+\mathrm{K}_{7} M+\frac{\mathrm{K}_{1} \mathrm{~K}_{5} \mathrm{~K}_{7} M e M}{\mathrm{~K}_{9} F}+\mathrm{K}_{9} F}$

$r_{9}=\frac{\mathrm{K}_{-9}\left(\frac{\mathrm{K}_{1} \mathrm{~K}_{2} \mathrm{~K}_{3} M e T}{\mathrm{~K}_{6} D}-\mathrm{K}_{9} F\right)}{1+\mathrm{K}_{1} M e+\mathrm{K}_{2} T+\mathrm{K}_{6} D+\mathrm{K}_{7} M+\mathrm{K}_{8} G+\frac{\mathrm{K}_{1} \mathrm{~K}_{2} \mathrm{~K}_{3} M e T}{\mathrm{~K}_{6} D}}$

$r_{1}=\frac{\mathrm{K}_{1}\left(M e-\frac{\mathrm{K}_{4} \mathrm{~K}_{5} G F}{\mathrm{~K}_{1} \mathrm{~K}_{2} \mathrm{~K}_{3} T}\right)}{1+\frac{\mathrm{K}_{4} \mathrm{~K}_{5} G F}{\mathrm{~K}_{2} \mathrm{~K}_{3} T}+\mathrm{K}_{2} T+\mathrm{K}_{4} G+\mathrm{K}_{5} F}$

$r_{2}=\frac{\mathrm{k}_{2}\left(T-\frac{\mathrm{K}_{4} \mathrm{~K}_{5} G F}{\mathrm{~K}_{1} \mathrm{~K}_{2} \mathrm{~K}_{3} M e}\right)}{1+\mathrm{K}_{1} M e+\frac{\mathrm{K}_{4} \mathrm{~K}_{5} G F}{\mathrm{~K}_{1} \mathrm{~K}_{3} M e}+\mathrm{K}_{4} G+\mathrm{K}_{5} F}$ 
Table 3: (continued)

\begin{tabular}{ll}
\hline Rate determining elementary step & Model \\
\hline$M e^{\star}+T^{\star} \rightleftharpoons G^{*}+F^{*}$ & $r_{3}=\frac{\mathrm{k}_{3}\left(\mathrm{~K}_{1} \mathrm{~K}_{2} M e T-\frac{\mathrm{K}_{4} \mathrm{~K}_{5} G F}{\mathrm{~K}_{3}}\right)}{\left(1+\mathrm{K}_{1} M e+\mathrm{K}_{2} T+\mathrm{K}_{4} G+\mathrm{K}_{5} F\right)^{2}}$ \\
$G^{*} \rightleftharpoons G+{ }^{*}$ & $r_{4}=\frac{\mathrm{k}_{-4}\left(\frac{\mathrm{K}_{1} \mathrm{~K}_{2} \mathrm{~K}_{3} M e T}{\mathrm{~K}_{5} F}-\mathrm{K}_{4} G\right)}{1+\mathrm{K}_{1} M e+\mathrm{K}_{2} T+\frac{\mathrm{K}_{1} \mathrm{~K}_{2} \mathrm{~K}_{3} M e T}{\mathrm{~K}_{5} F}+\mathrm{K}_{5} F}$ \\
$F^{*} \rightleftharpoons F^{*}$ & $r_{5}=\frac{\mathrm{K}_{-5}\left(\frac{\mathrm{K}_{1} \mathrm{~K}_{2} \mathrm{~K}_{3} M e T}{\mathrm{~K}_{4} G}-\mathrm{K}_{5} F\right)}{1+\mathrm{K}_{1} M e+\mathrm{K}_{2} T+\mathrm{K}_{4} G+\frac{\mathrm{K}_{1} \mathrm{~K}_{2} \mathrm{~K}_{3} M e T}{\mathrm{~K}_{4} G}}$
\end{tabular}

(c) Based on 7 elementary steps ER mechanism

$$
\begin{aligned}
& M e{ }^{*} \rightleftharpoons M e^{\star} \\
& M e^{\star}+T \rightleftharpoons D^{\star}+F \\
& M e^{\star}+D \rightleftharpoons M^{\star}+F \\
& M e^{\star}+M \rightleftharpoons G^{\star}+F \\
& D^{\star} \rightleftharpoons D+{ }^{*} \\
& M^{\star} \rightleftharpoons M+{ }^{*} \\
& G^{\star} \rightleftharpoons G+{ }^{*}
\end{aligned}
$$

$$
\begin{aligned}
& r_{1}=\frac{\mathrm{k}_{1}\left(M e-\frac{\mathrm{K}_{5} D F}{\mathrm{~K}_{1} \mathrm{~K}_{2} T}\right)}{1+\frac{\mathrm{K}_{5} D F}{\mathrm{~K}_{2} T}+\mathrm{K}_{5} D+\mathrm{K}_{6} M+\mathrm{K}_{7} G} \\
& r_{2}=\frac{\mathrm{K}_{2}\left(\mathrm{~K}_{1} M e T-\frac{\mathrm{K}_{5} D F}{\mathrm{~K}_{2}}\right)}{1+\mathrm{K}_{1} M e+\mathrm{K}_{5} D+\mathrm{K}_{6} M+\mathrm{K}_{7} G} \\
& r_{3}=\frac{\mathrm{K}_{3}\left(\mathrm{~K}_{1} M e D-\frac{\mathrm{K}_{6} M F}{\mathrm{~K}_{3}}\right)}{1+\mathrm{K}_{1} M e+\mathrm{K}_{5} D+\mathrm{K}_{6} M+\mathrm{K}_{7} G} \\
& r_{4}=\frac{\mathrm{K}_{4}\left(\mathrm{~K}_{1} M e M-\frac{\mathrm{K}_{7} G F}{\mathrm{~K}_{4}}\right)}{1+\mathrm{K}_{1} M e+\mathrm{K}_{5} D+\mathrm{K}_{6} M+\mathrm{K}_{7} G} \\
& r_{5}=\frac{\mathrm{K}_{-5}\left(\frac{\mathrm{K}_{1} \mathrm{~K}_{2} T M e}{F}-\mathrm{K}_{5} D\right)}{1+\mathrm{K}_{1} M e+\frac{\mathrm{K}_{1} \mathrm{~K}_{2} T M e}{F}+\mathrm{K}_{6} M+\mathrm{K}_{7} G} \\
& r_{6}=\frac{\mathrm{k}_{-6}\left(\frac{\mathrm{K}_{1} \mathrm{~K}_{3} D M e}{F}-\mathrm{K}_{6} M\right)}{1+\mathrm{K}_{1} M e+\mathrm{K}_{5} D+\frac{\mathrm{K}_{1} \mathrm{~K}_{3} D M e}{F}+\mathrm{K}_{7} G} \\
& r_{7}=\frac{\mathrm{k}_{-7}\left(\frac{\mathrm{K}_{1} \mathrm{~K}_{4} M M e}{F}-\mathrm{K}_{7} G\right)}{1+\mathrm{K}_{1} M e+\mathrm{K}_{5} D+\mathrm{K}_{6} M+\frac{\mathrm{K}_{1} \mathrm{~K}_{4} M M e}{F}}
\end{aligned}
$$

(d) Based on 3 elementary steps ER mechanism

$$
\begin{aligned}
& M e{ }^{*} \rightleftharpoons M e^{\star} \\
& M e^{\star}+T \rightleftharpoons G^{\star}+F \\
& G^{\star} \rightleftharpoons G{ }^{*}
\end{aligned}
$$$$
\begin{aligned}
& r_{1}=\frac{\mathrm{k}_{1}\left(M e-\frac{\mathrm{K}_{3} G F}{\mathrm{~K}_{1} \mathrm{~K}_{2} T}\right)}{1+\frac{\mathrm{K}_{3} G F}{\mathrm{~K}_{2} T}+\mathrm{K}_{3} G} \\
& r_{2}=\frac{\mathrm{k}_{2}\left(\mathrm{~K}_{1} M e T-\frac{\mathrm{K}_{3} G F}{\mathrm{~K}_{2}}\right)}{1+\mathrm{K}_{1} M e+\mathrm{K}_{3} G} \\
& r_{3}=\frac{\mathrm{k}_{-3}\left(\frac{\mathrm{K}_{1} \mathrm{~K}_{2} T M e}{\mathrm{~F}}-\mathrm{K}_{3} G\right)}{1+\mathrm{K}_{1} M e+\frac{\mathrm{K}_{1} \mathrm{~K}_{2} T M e}{F}}
\end{aligned}
$$

adsorption and desorption the $\mathrm{K}$ letters represent the equilibrium adsorption and desorption constants respectively while in the elementary steps of reaction surface they represent the kinetic constants at equilibrium.

The model discrimination was based on the physical significance of the estimated parameters and on the comparison between the experimental reaction rates and the calculated rates from the proposed models. These rates were calculated by estimating the corresponding parameters first. Models were rejected if showed a different trend from the experimental data or kinetic parameters with erroneous values, i. e., negative values, too high values or not significantly different from zero at the $95 \%$ confidence level.

The rejected models were all based on LHHW or EleyRideal mechanisms with three surface reactions with formation of glycerides and monoglycerides as intermediaries. This is consistent with the concentration data of intermediate compounds shown in Figure 4. The concentration of diglycerides and monoglycerides in the liquid phase of the reaction mixture shows no significant change compared to triglycerides and methyl esters with 
reaction time. Because the ester groups of the triglycerides are less susceptible to be attacked by the active species formed by the adsorbed methanol than those in diglycerides and monoglycerides, due to steric hindrance caused by the long-chain fatty acids, the presence of intermediates in the reaction bulk is lower, because once formed react quickly with adsorbed methanol onto the catalytic surface until be transformed into methyl esters, as is suggested by Likozar, and Levec (2014). It can be inferred from the obtained methyl esters content that nor diglycerides or monoglycerides remain chemisorbed onto the catalytic surface. This assumption is based upon comparison of the number of moles of methyl esters experimentally observed with the expected from the theoretical stoichiometric analysis. This comparison is shown in Figure 3. This concentration profile is similar to that obtained in kinetic studies using homogeneous catalysts, in which one reaction is considered without the formation of intermediate compounds (Stamenković et al. 2008, Stamenković et al. 2010). Under this assumption, the transesterification reaction using $\mathrm{K}_{2} \mathrm{O} / \mathrm{NaX}$ catalyst seems plausible to occur via a single surface reaction mechanism. Actually, based on the estimated parameters, confidence intervals and regression coefficients for all models, it can be concluded that regardless the mechanism, assuming the surface reaction as determining step leads to either a poor experimental data fitting or to negative values of the estimated parameters. It can also be concluded that when assuming the surface reaction as limiting step, the LHHW mechanism based models are less precise than those based on ER mechanism. It is also worth pointing out that albeit the high regression coefficient the reaction rate expressions obtained by assuming the desorption step was limiting were discarded because either the parameters were negative or the confidence intervals were rather large.

From LHHW and Eley-Rideal mechanisms with only one surface reaction without intermediate compounds, two models with the same trend as the experimental data and significant parameters were established. These models assume methanol adsorption as limiting step. The LHHW (model A) and ER (model B) reaction rate equations are eqs (4) and (5), respectively. The estimated parameters via non-linear regression of eqs (4) and (5) are shown in Table 4.

$$
\begin{gathered}
r_{A}=\frac{\mathrm{k}_{1}\left(M e-\frac{\mathrm{K}_{4} \mathrm{~K}_{5} G F}{\mathrm{~K}_{1} \mathrm{~K}_{2} \mathrm{~K}_{3} T}\right)}{1+\frac{\mathrm{K}_{4} \mathrm{~K}_{5} G F}{\mathrm{~K}_{2} \mathrm{~K}_{3} T}+\mathrm{K}_{2} T+\mathrm{K}_{4} G+\mathrm{K}_{5} F} \\
r_{B}=\frac{\mathrm{k}_{1}\left(M e-\frac{\mathrm{K}_{3} G F}{\mathrm{~K}_{1} \mathrm{~K}_{2} T}\right)}{1+\frac{\mathrm{K}_{3} G F}{\mathrm{~K}_{2} T}+\mathrm{K}_{3} G}
\end{gathered}
$$

The residual sum of squares (RSS) of model B is smaller than the RSS of model A. This indicates that Model B better describes the experimental data than Model A. Furthermore, the adsorption equilibrium constant of methanol $\left(K_{1}\right)$ in model A, is several orders of magnitude greater than the adsorption equilibrium constant of triglycerides. This does not correspond to the methanol adsorption being the determining step. Triglycerides are larger molecules and less polar than methanol. This can hinder their adsorption on the catalyst active sites,

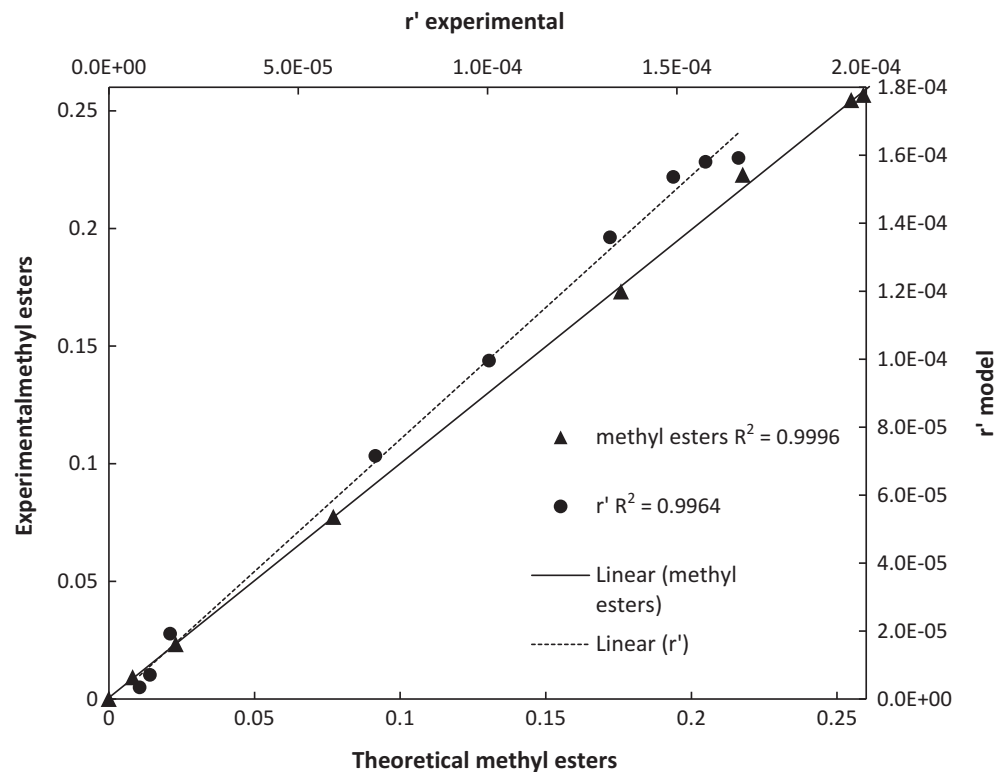

Figure 3: Parity plot of experimental and theoretical methyl esters content and reaction rates obtained experimentally and by the model ER reaction with one surface reaction and assuming the absorption of methanol as a determining step. 
Table 4: Parameter estimates with their $95 \%$ confidence interval.

\begin{tabular}{|c|c|c|c|c|c|}
\hline \multicolumn{3}{|l|}{ Model A } & \multicolumn{3}{|l|}{ Model B } \\
\hline Variable & Value & $95 \%$ confidence & Variable & Value & $95 \%$ confidence \\
\hline$k$ & 0.0001008 & $3.58 \mathrm{E}-07$ & $k$ & $9.65 \mathrm{E}-05$ & $3.86 \mathrm{E}-08$ \\
\hline$K_{1}$ & 2.009943 & 0.0510239 & $\kappa_{1}$ & 10.08985 & 0.0954457 \\
\hline$K_{2}$ & 0.0746827 & 0.0015881 & $K_{2}$ & 11.15178 & 0.0380413 \\
\hline$K_{3}$ & 19.72865 & 0.4195795 & $K_{3}$ & 4.693079 & 0.0108143 \\
\hline$K_{4}$ & 3.036386 & 0.0425042 & & & \\
\hline$K_{5}$ & 0.0592703 & 0.0012587 & & & \\
\hline Precision & & & Precision & & \\
\hline$R^{2}$ & & 0.988384 & $R^{2}$ & & 0.9962207 \\
\hline$R_{a d j}^{2}$ & & 0.9690239 & $R_{a d j}^{2}$ & & 0.9939531 \\
\hline$R_{m s d}$ & & $2.23 \times 10^{-6}$ & $R_{m s d}$ & & $1.27 \times 10^{-6}$ \\
\hline$\sigma^{2}$ & & $1.34 \times 10^{-10}$ & $\sigma^{2}$ & & $2.61 \times 10^{-11}$ \\
\hline$x^{2}$ & & $2.40899 \times 10^{-5}$ & $x^{2}$ & & $5.18152 \times 10^{-6}$ \\
\hline RSS & & $4.0176 \times 10^{-10}$ & RSS & & $1.30707 \times 10^{-10}$ \\
\hline
\end{tabular}

causing their adsorption being the determining step. The model that assumes this was not satisfactory though. The discrimination parameter $P_{A, B}$ was calculated to select the best model between model $\mathrm{A}$ and $\mathrm{B}$ and $\mathrm{a}$ value of 7.7487 was obtained. This suggests that it is more plausible that model $\mathrm{B}$ better describes the experimental data. The comparison of experimental reaction rates and those calculated with model $B$ is shown in Figure 3. The evolution of mol number of reactants and products obtained with the model are shown in Figure 4.

The adsorption of methanol as limiting step has also been proposed by Dossin, Reyniers, and Marin 2006 and
Kapil et al. 2011. This is also in agreement with the reaction mechanism proposed by Kusuma et al. 2013, wherein has been postulated that without the formation of the methoxide compound in the active sites of $\mathrm{K}_{2} \mathrm{O}$ in the zeolite, the transesterification reaction does not start.

It is also worth pointing out that the above mentioned models were established with fresh catalysts. It has been recently reported (Muciño et al. 2015), however, that this catalyst deactivates mainly by leaching and the deactivation law was determined to be second order. This should be bare on mind in order to obtain a more accurate model.

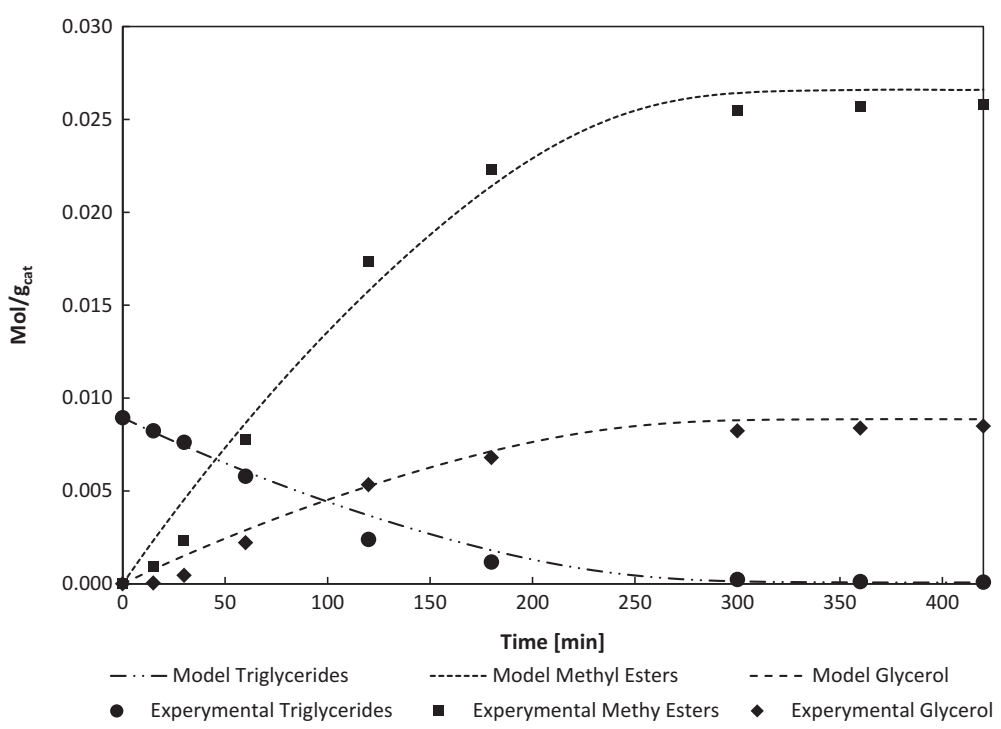

Figure 4: Experimental and predicted moles of reactants and products by ER Mechanism (Model B). Molar ratio of methanol to oil $18: 1$. Temperature: $60^{\circ} \mathrm{C}$. Amount of catalyst: $10 \mathrm{wt} \%$. 


\section{Conclusions}

A kinetic model that describes the catalyzed transesterification reaction of safflower oil with methanol was obtained. This model is based on an Eley-Rideal type chemical mechanism that assumes the methanol adsorption as limiting step. Accordingly, it can be postulated that triglycerides do not adsorb in order to react as evidenced by the low fitting obtained with LHHW models when assuming reagents adsorption at the surface and surface reaction as determining steps. Therefore, saturating the catalytic surface with methanol at high molar methanol:oil ratios will not inhibit but will promote the transesterification reaction of safflower oil.

Funding: The authors are grateful to Universidad Autónoma del Estado de México for financial support through Project 3685/2014CIB. G.E.G. wishes to thank Consejo Nacional de Ciencia y Tecnología (CONACYT) for scholarship No.266962 to conduct postgraduate studies, to Facultad de Ciencias y Tecnologías Químicas and the Instituto de Tecnología Química y Medioambiental (ITQUIMA) of Universidad de Castilla-La Mancha for their collaboration in the project.

\section{References}

1. Cao, F., Chen, Y., Zhai, F., Li, J., Wang, J., Wang, X., Wang, S., Zhu, W., 2008. Biodiesel production from high acid value waste frying oil catalyzed by superacid heteropolyacid. Biotechnology and Bioengineering 101, 93-100.

2. Câmara, L.D.T., Aranda, D.A.G., 2011. Reaction kinetic study of biodiesel production from fatty acids esterification with ethanol. Industrial and Engineering Chemistry Research 50, 2544-2547.

3. Demirbas, A., 2008a. Biodiesel A Realistic Fuel Alternative for Diesel Engines, 1st ed, Springer-Verlag, London.

4. Demirbas, A., 2008b. Comparison of transesterification methods for production of biodiesel from vegetable oils and fats. Energy Conversion and Management 49, $125-130$.

5. Dossin, T.F., Reyniers, M.F., Marin, G.B., 2006. Kinetics of heterogeneously MgO-catalyzed transesterification. Applied Catalysis B: Environmental 62, 35-45.

6. Froment, G.F., Bischoff, K.B., De Wilde, J., 2011. Chemical Reactor Analysis and Design, 3rd Ed, Wiley, EUA.

7. Gerpen, J.V., 2005. Biodiesel processing and production. Fuel Processing Technology 86, 1097-1107.

8. Granados, M.L., Martín Alonso, D., Sádaba, I., Mariscal, R., Ocón, P., 2009. Leaching and homogeneous contribution in liquid phase reaction catalysed by solids: The case of triglycerides methanolysis using $\mathrm{CaO}$. Applied Catalysis B: Environmental 89, 265-272.
9. Hattori, H., Shima, M., Kabashima, H., 2000. Alcoholysis of ester and epoxide catalyzed by solid bases, in Elsevier (Ed), Studies in Surface Science and Catalysis 12th International Congress on Catalysis, 3507-3512. Spain.

10. Kapil, A., Wilson, K., Lee, A.F., Sadhukhan, J., 2011. Kinetic Modeling Studies of Heterogeneously Catalyzed Biodiesel Synthesis Reactions. Industrial \& Engineering Chemistry Research 50, 4818-4830.

11. Kawashima, A., Matsubara, K., Honda, K., 2009. Acceleration of catalytic activity of calcium oxide for biodiesel production. Bioresource Technology 100, 696-700.

12. Kim, H., Kang, B., Kim, M., Park, Y., Kim, D., Lee, J., Lee, K., 2004. Transesterification of vegetable oil to biodiesel using heterogeneous base catalyst. Catalysis Today 93-95, 315-320.

13. Kusuma, R.I., Hadinoto, J.P., Ayucitra, A., Soetaredjo, F.E., Ismadji, S., 2013. Natural zeolite from Pacitan Indonesia, as catalyst support for transesterification of palm oil. Applied Clay Science 74, 121-126.

14. Lee, D., Park, Y., Lee, K., 2009. Heterogeneous Base Catalysts for transesterification in biodiesel Synthesis. Catalysis Surveys from Asia 13, 63-77.

15. Likozar, B., Levec, J., 2014. Effect of process conditions on equilibrium, reaction kinetics and mass transfer for triglyceride transesterification to biodiesel: Experimental and modeling based on fatty acid composition. Fuel Processing Technology 122, 30-41.

16. Math, M.C., Kumar, S.P., Chetty, S.V., 2010. Technologies for biodiesel production from used cooking oil-a review. Energy for Sustainable Development 14, 339-345.

17. Meher, L.C., Viday, S.D., Naik, S.N., 2006. Technical aspects of biodiesel production by transesterification-a review. Renewable and Sustainable Energy Reviews 10, 248-268.

18. Meneghetti, S.M.P., Meneghetti, M.R., Serra, T.M., Barbosa, D.C., Wolf, C.R., 2007. Biodiesel production from vegetable oil mixtures: Cottonseed, soybean, and castor oils. Energy \& Fuels 21, 3746-3747.

19. Muciño, G.G., Romero, R., García-Orosco, I., Ramírez, A., Baeza-Jiménez, R., Natividad, R., In Press. Deactivation study of $\mathrm{K}_{2} \mathrm{O} / \mathrm{NaX}$ and $\mathrm{Na}_{2} \mathrm{O} / \mathrm{NaX}$ catalysts for biodiesel production. Catalysis Today.

20. Peña, R.. 2009. Obtención de biodiesel partir de aceites vegetales utilizando catalizadores heterogéneos de oxido de magnesio y nitrato de potasio. Maestría En Ciencias Químicas, Ingeniería Química, Universidad Autónoma del Estado de México.

21. Peña, R., Romero, R., Martínez, S.L., Natividad, R., Ramírez, A., 2013. Characterization of $\mathrm{KNO}_{3} / \mathrm{NaX}$ catalyst for sunflower oil transesterification. Fuel 110, 63-69.

22. Stamenković, O.S., Todorović, Z.B., Lazić, M.L., Veljković, V.B., Skala, D.U., 2008. Kinetics of sunflower oil methanolysis at low temperatures. Bioresource Technology 99, 1131-1140.

23. Stamenković, O.S., Veljković, V.B., Todorović, Z.B., Lazić, M.L., Banković-Ilić, I.B., Skala, D.U., 2010. Modeling the kinetics of calcium hydroxide catalyzed methanolysis of sunflower oil. Bioresource Technology 101, 4423-4430. 
24. Van de Steene, E., De Clercq, J., Thybaut, J.W., 2012. Adsorption and reaction in the transesterification of ethyl acetate with methanol on Lewatit K1221. Journal of Molecular Catalysis A: Chemical 359 , 57-68.

25. Venkat Reddy, C.R., Oshel, R., Verkade, J.G., 2006. RoomTemperature conversion of coybean oil and poultry fat to biodiesel catalyzed by nanocrystalline calcium oxides. Energy \& Fuels 20, 1310-1314.
26. Zabeti, M., Wan Daud, W.M.A., Aroua, M.K., 2009. Activity of solid catalysts for biodiesel production: a review. Fuel Processing Technology 90, 770-777.

27. Zhang, J., Chen, S., Yang, R., Yan, Y., 2010. Biodiesel production from vegetable oil using heterogenous acid and alkali catalyst. Fuel 89, 2939-2944.

28. Zhou, W., Boocock, D., 2006. Phase behavior of the basecatalyzed transesterification of soybean oil. Journal of the American Oil Chemists' Society 83, 1041-1045. 